\title{
Trends in infection-related hospital admissions and impact of length of time on dialysis among patients on long-term dialysis: a retrospective cohort study
}

\author{
Jean-Philippe Lafrance MD MSc, Elham Rahme PhD, Sameena Iqbal MD, Naoual Elftouh MSc, \\ Louis-Philippe Laurin MD, Michel Vallée MD PhD
}

\section{Abstract}

Background: After cardiovascular disease, infection is the second leading reason for admission to hospital among patients receiving long-term dialysis. We examined whether duration of dialysis treatment influences the rate of infection-related admission to hospital.

Methods: Using provincial administrative databases for Quebec, we built a retrospective cohort of all adults receiving long-term dialysis (hemodialysis or peritoneal dialysis) between 2001 and 2007. We evaluated rates of infection-related admission to hospital according to length of time on dialysis.

Results: A cohort of 9822 patients (mean age 66.3 [standard deviation \pm 14.7 ] yr; $39.7 \%$ female) were followed for a median of 2.1 (range 1.0-3.9) years. Between 2001 and 2007, infection-related hospital admissions remained stable (from 0.20 to 0.19 per person-year; $p=0.7$ ). All-cause hospital admission rates decreased by $22.9 \%$ (from 1.53 to 1.18 per person-year; $p<0.001$ ), and cardiovascularrelated admission rates decreased by $46.7 \%$ (from 0.45 to 0.24 per person-year; $p<0.001$ ). The rate of infection-related admission remained stable with increasing time on dialysis $(p=0.1)$; however, both all-cause and cardiovascular-related admission rates decreased with length of time on dialysis $(p<0.001)$. Standardization of hospital admission rates by age, sex or length of time on dialysis did not change trends.

Interpretation: We found a stable rate of infection-related hospital admission between 2001 and 2007 among patients on long-term dialysis, independent of age, sex and length of time on dialysis. A decrease in all-cause and cardiovascular-related admission rates during the same period meant that the proportion of admissions related to infection increased. Because admissions to hospital are potentially preventable, understanding the epidemiology of infection-related admissions may inform future studies on prevention of this serious outcome.

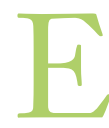
nd-stage renal disease is the last stage of kidney failure, and it is irreversible. To survive, patients require renal replacement therapy: either dialysis (hemodialysis or peritoneal dialysis) or kidney transplantation. Long-term dialysis is associated with a high burden of morbidity and frequent admission to hospital. ${ }^{1,2}$ Studies conducted in the United States have reported high rates of infection-related admissions to hospital and associated morbidity and mortality in the dialysis population. ${ }^{1,3-7}$ Although rates of all-cause admissions to hospital remained fairly stable between 1994 and 2009 in the US, rates of infection-related admissions among patients on dialysis increased by $30.6 \%$. $^{1}$ These findings led to a recognition of the need to decrease the use of tunnelled hemodialysis catheters, one of the main causes of infection in this population. ${ }^{1,8}$ Similar studies have not been conducted in Canada, and US estimates may not apply to the Canadian population because dialysis populations differ substantially between the 2 countries $^{1,8}$ in terms of demographics, ${ }^{1,8,9}$ use of vascular access ${ }^{10}$ and mortality rates, ${ }^{1,8}$ among other factors.
Because mortality is increased among patients with major comorbidities and older patients, those who have survived a few years of dialysis differ greatly from patients initiating dialysis. Although one might hypothesize that the rate of admission to hospital would decrease with time on dialysis, other factors come into play: greater use of catheters in the early stages of dialysis, cardiovascular and other complications and kidney transplant. In brief, how rates of infection-related admissions to hospital vary with length of time on dialysis remains unpredictable and not well described. Because the proportion of new

Competing interests: None declared.

This article has been peer reviewed.

Correspondence to: Jean-Philippe Lafrance, jean-philippe.lafrance@ umontreal.ca

CMAJ Open 2014.DOI:10.9778/cmajo.20120027 
and long-term dialysis patients may change over time, reporting rates of hospital admission without controlling for length of time on dialysis may confound results. Until now, trends in rates of infection-related hospital admissions have usually been reported only for long-term dialysis patients. The US Renal Data System's 2011 annual report presented data on new dialysis patients, but only rates of admission in the first year were reported. ${ }^{1}$ Because infection-related hospital admissions are potentially preventable, it is important to assess their frequency and associated risk factors to inform prevention strategies aimed at improving patient care.

We conducted this study to describe population-based trends in the incidence of infection-related hospital admissions among new and long-term dialysis patients from 2001 to 2007 , with a focus on length of time on dialysis.

\section{Methods}

\section{Data sources}

We obtained data from health services administrative databases in the province of Quebec, Canada. The physician and hospital services of virtually all 8 million residents in Quebec are covered by a universal single payer: the Régie de l'assurance maladie du Québec. The physician fee-for-service claims database includes all visits, diagnostic codes and procedures during inand outpatient encounters. Primary diagnoses (coded by medical archivists using the International Classification of Diseases, 9th Revision [Clinical Modification] [ICD-9] or the International Statistical Classification of Diseases and Related Health Problems, 10th revision [ICD-10] after 2006) for each admission to hospital were obtained from the hospital discharge summary database. Discharge summary data in Quebec have been validated for a number of conditions, including hip fracture, ${ }^{11}$ stroke, ${ }^{12}$ myocardial infarction ${ }^{13}$ and injuries. ${ }^{14}$ For these conditions, data accuracy was generally high.

\section{Study cohort}

The study cohort included all adults (age $\geq 18 \mathrm{yr}$ ) who were on dialysis (hemodialysis or peritoneal dialysis) between Jan. 1, 2001, and Dec. 31, 2007. Patients were identified using physician claims or admission discharge sheets. Both new patients initiating dialysis during the study period and patients already on dialysis at the beginning of the study were included. Although limiting the cohort to new patients would have resulted in a simpler cohort, including patients who had been on dialysis for varying lengths of time as of 2001 was essential to evaluate trends over the entire study period. Moreover, in other dialysis registries, hospital admission rates are usually reported for the long-term dialysis population. Finally, one of our objectives was to describe the burden of infection-related admissions to hospital in the whole dialysis population during the study period. We excluded patients who had less than 90 days of follow-up after initiation of dialysis (due to death, recovery of kidney function or dialysis started within 90 days of the end of the study) and those who had a prior kidney transplant (Figure 1). The 90-day criterion was used to ensure that only maintenance dialysis patients were included and to ensure that our patient population was comparable to other long-term dialysis cohorts. Patients were followed from Jan. 1, 2001, or dialysis initiation after that date until death, kidney transplantation or the end of the study (Dec. 31, 2007).

\section{Admission categories}

We identified all admissions to hospital during the study period, excluding those required for dialysis initiation. Each admission was categorized using ICD-9 or ICD-10 codes corresponding to the primary diagnosis on the discharge sheet grouped into 3 mutually exclusive categories: infection, cardiovascular disease or other cause (see eTable 1 in Appendix 1 for specific codes). Categories and codes were based on US Renal Data System methods to allow comparison between populations. ${ }^{1}$ Although the focus of this study was hospital admissions related to infection, we also included cardiovascular disease because it is the principal cause of admissions in this population. Infections were subdivided into dialysis-related infections, pneumonia, septicemia and other infections.

\section{Length of time on dialysis}

Length of time on dialysis was calculated for each patient for every calendar year and categorized as less than 1 year, 1 to less than 2 years, 2 to less than 3 years, etc. The length of time for patients initiating dialysis in the current calendar year was classified as less than 1 year. Because we had no access to data before Jan. 1, 1999, patients with dialysis codes on that date were assumed to have started dialysis in 1998 or earlier. These patients were categorized as having a length of time on dialysis of at least 3 years in 2001, at least 4 years in 2002, at least 5 years in 2003, and so on.

\section{Comorbidities}

Comorbidities were assessed at the beginning of the study period using ICD-9 codes for diagnoses in the 2 years before the starting date. They included cardiovascular disease, cerebrovascular disease, congestive heart failure, chronic pulmonary disease, diabetes, malignant disease and peripheral vascular disease (see e'Table 2 in Appendix 1 for codes).

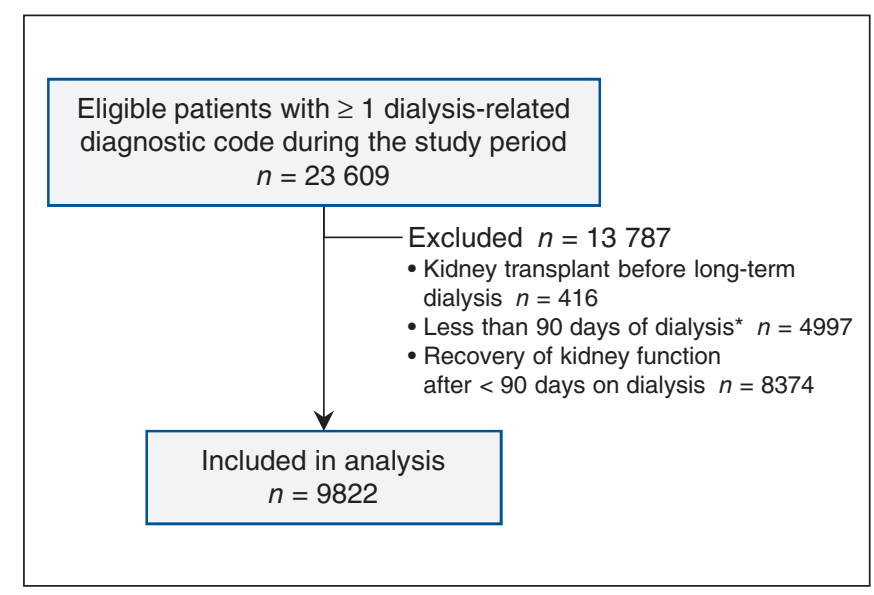

Figure 1: Selection of study cohort. *Because of death or because dialysis started less than 90 days from the end of the study period. 


\section{Statistical analysis}

We calculated unadjusted admission rates by dividing the number of admissions by the total number of patient-years (i.e., the number of days from dialysis initiation to the end of follow-up for all patients divided by $365.25 \mathrm{~d}$ ). The $95 \%$ confidence intervals for admission rates were calculated with the use of a Poisson distribution with a robust error variance to account for clustering of data. ${ }^{15}$ We tested linear trends using the Mantel-Haenszel trend statistic. ${ }^{16}$ We produced rates of admission adjusted for length of time on dialysis by applying the stratified rates for lengths of time on dialysis $(<1,1,2$, $\geq 3 \mathrm{yr}$ ) that were common for all calendar years to the persontime distribution of the whole cohort (direct standardization). ${ }^{17}$ Similarly, we calculated age- and sex-standardized rates using the same reference population and 4 age strata $(<55,55-64$, $65-74, \geq 75 \mathrm{yr}$ ). Because of the prohibitive number of strata, we were not able to calculate adjusted rates for both length of time on dialysis and demographics. However, we evaluated statistical interactions between length of time on dialysis and calendar time by including a multiplicative term in a Poisson multivariable regression model with a robust error variance.

In a sensitivity analysis, we redid all analyses excluding patients who started dialysis before January 1999, because their initiation date was unknown. Similarly, we conducted an analysis limited to patients initiating dialysis during the study period. Finally, all analyses were redone by dialysis modality.

\section{Ethics approval}

This study was approved by the Maisonneuve-Rosemont Hospital and Government of Quebec (Commission d'accès à l'information) ethics committees, and informed consent was waived.

\section{Results}

Baseline characteristics of the 9822 patients in the study cohort are shown in Table 1. The mean age was 66.3 (standard deviation \pm 14.7 ) years, $39.7 \%$ were women, and $81.0 \%$ were on hemodialysis. Diabetes and cardiovascular disease were the most prevalent comorbidities ( $45.1 \%$ and $41.3 \%$, respectively). Patients newly started on dialysis $(n=6567)$ represented $66.9 \%$ of the cohort.

Patients were followed for a median time of 2.1 years (interquartile range [IQR] 1.0-3.9 yr). During the study period, we identified 35246 admissions to hospital. Recurrent admissions for a single patient were common: no admissions, 1181 patients (12.0\%); 1 admission, 1923 patients (19.6\%); 2 admissions, 1645 patients (16.7\%); 3 or more admissions, 5073 patients $(51.6 \%)$. The median length of stay in hospital was 4 days (IQR 1-12 d). Rates of admission are shown in Table 2. As expected, cardiovascular-related admissions were the most frequent ( 0.354 per person-year), followed by infection-related admissions ( 0.200 per person-year). Among infection-related admissions, those associated with dialysis were the most frequent ( 0.046 per person-year).

Trends in admission rates over time are shown in Table 3 and Figure 2. Between 2001 and 2007, the rate of infectionrelated hospital admissions remained stable (Mantel-Haenszel trend test $p=0.7$ ). During the same period, all-cause admission rates decreased by $22.9 \%$ (from 1.53 to 1.18 per person-year; $p<0.001$ ); therefore, the proportion of admissions related to infection increased from $13.1 \%$ to $16.1 \%$ of all admissions. Cardiovascular-related admission rates decreased by $46.7 \%$ (from 0.45 to 0.24 per person-year; $p<0.001$ ).

As shown in Figure 3, infection-related hospital admissions remained fairly stable regardless of length of time on dialysis

Table 1: Baseline characteristics of the study population $(n=9822)$

\begin{tabular}{|c|c|}
\hline Characteristic & No. $(\%)^{*}$ \\
\hline Age, yr, mean $\pm S D$ & $66.3 \pm 14.7$ \\
\hline Sex, female & $3896(39.7)$ \\
\hline \multicolumn{2}{|l|}{ Dialysis type $†$} \\
\hline Hemodialysis & $7958(81.0)$ \\
\hline Peritoneal dialysis & $1864(19.0)$ \\
\hline \multicolumn{2}{|l|}{ Duration of dialysis } \\
\hline New & $6567(66.9)$ \\
\hline Long-term & $3255(33.1)$ \\
\hline \multicolumn{2}{|c|}{ Admissions to hospital in previous year } \\
\hline 0 & $4595(46.7)$ \\
\hline 1 & $2671(27.2)$ \\
\hline$\geq 2$ & $2556(26.0)$ \\
\hline \multicolumn{2}{|l|}{ Comorbidities } \\
\hline Diabetes & $4431(45.1)$ \\
\hline Cardiovascular disease & $4055(41.3)$ \\
\hline Congestive heart failure & $2480(25.2)$ \\
\hline Peripheral vascular disease & $2378(24.2)$ \\
\hline Chronic pulmonary disease & $1639(16.7)$ \\
\hline Malignant disease & $1265(12.9)$ \\
\hline Cerebrovascular disease & $697(7.1)$ \\
\hline $\begin{array}{l}\text { Note: } \mathrm{SD}=\text { standard deviation. } \\
{ }^{*} \text { Except where noted. } \\
\text { †Assigned at cohort entry and not up }\end{array}$ & \\
\hline
\end{tabular}

Table 2: Causes and rates of admission to hospital among patients on dialysis $(n=9822), 2001-2007$

\begin{tabular}{|lcc|}
\hline Cause & $\begin{array}{c}\text { No. of } \\
\text { admissions }\end{array}$ & $\begin{array}{c}\text { Rate per person-year* } \\
(95 \% \mathrm{Cl})\end{array}$ \\
\hline All & 35246 & $1.35(1.29-1.42)$ \\
\hline Cardiovascular disease & 9240 & $0.35(0.33-0.38)$ \\
\hline Infection & 5209 & $0.20(0.19-0.21)$ \\
\hline Dialysis-related & 1196 & $0.05(0.04-0.06)$ \\
\hline Pneumonia & 734 & $0.03(0.03-0.03)$ \\
\hline Septicemia & 578 & $0.02(0.02-0.03)$ \\
\hline Other infection & 2701 & $0.10(0.09-0.12)$ \\
\hline Other & 20797 & $0.80(0.77-0.83)$ \\
\hline $\begin{array}{l}\text { Note: Cl = confidence interval. } \\
\text { *Calculated as no. of days from dialysis initiation to the end of follow-up for all } \\
\text { patients divided by 365.25 days. }\end{array}$ \\
\hline
\end{tabular}




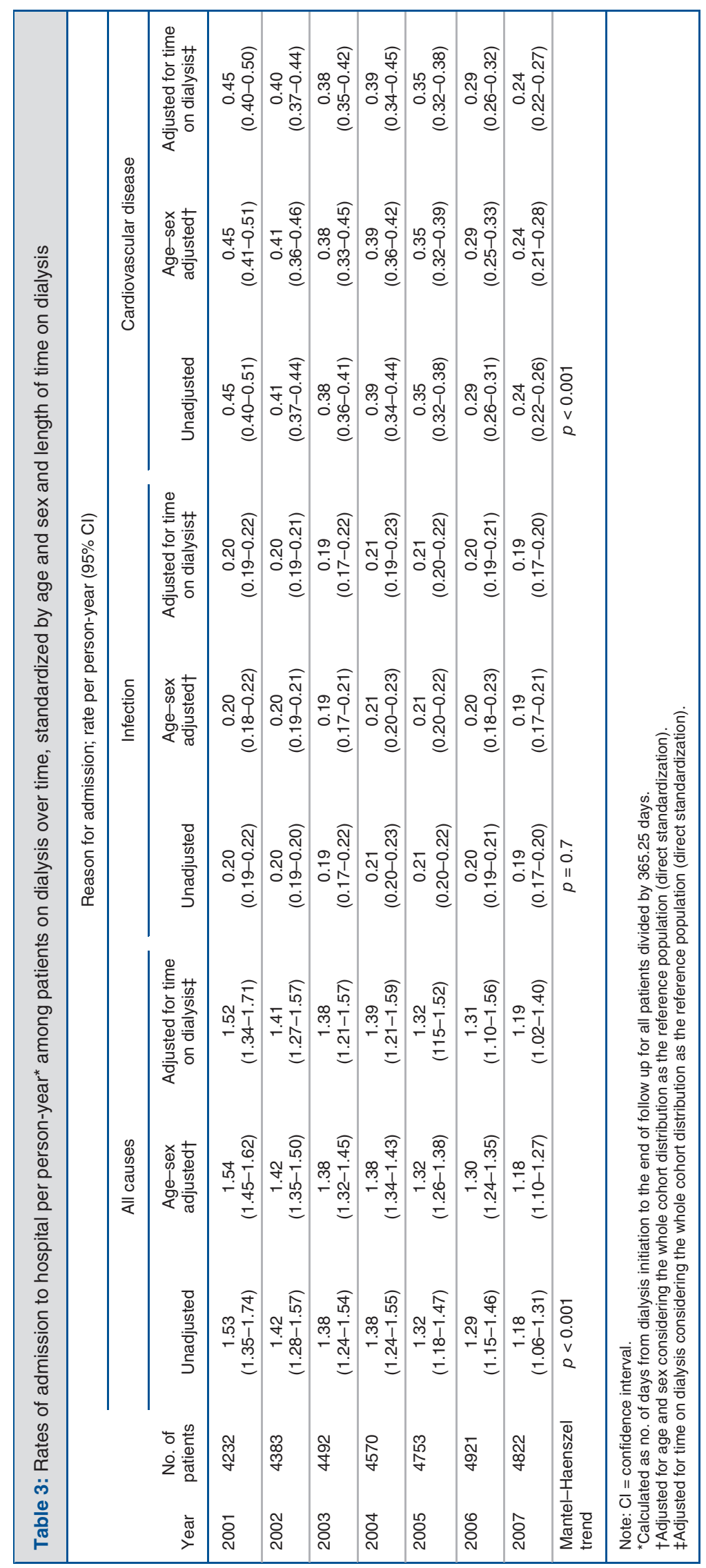


$(p=0.1)$. In contrast, all-cause and cardiovascular-related admission rates decreased with time on dialysis $(p<0.001$ for both), and the decrease was most pronounced during the first 3 years after dialysis initiation.

Interaction between calendar time and length of time on dialysis was not statistically significant for infection-related $(p=0.8)$ or cardiovascular-related $(p=0.09)$ admissions to hospital. However, it was statistically significant for all-cause rates of admission $(p=0.03)$; that is, trends in rates of admission varied over time for different categories of time on dialysis. However, rates standardized for length of time on dialysis were highly similar to unadjusted rates (Table 3), which indicated that this interaction had little influence on trends. Age- and sex-adjusted rates were also close to unadjusted rates (Table 3).

The sensitivity analyses, in which we excluded patients who had started dialysis before January 1999 or excluded prevalent patients, led to similar results (data not shown). Trends for hemodialysis and peritoneal dialysis patients were similar (data not shown).

\section{Interpretation}

This study describes trends in infection-related hospital admissions and the impact of length of time on dialysis in a large cohort of Canadian patients on long-term dialysis. Although infection-related admission rates were stable, rates of all-cause and cardiovascular-related admissions decreased over time. Therefore, the hospital burden attributable to infection increased over the years. Similarly, length of time on dialysis was associated with decreasing rates of all-cause and cardiovascular-related admissions, but not with infectionrelated admissions. Although we found an interaction between calendar time and length of time on dialysis for all-cause hospital admissions, trends in hospital admission rates adjusted for length of time on dialysis were similar to unadjusted rates.

\section{Comparison with other studies}

All-cause and infection-related hospital admission rates among patients on dialysis have not been studied in Canada. The 2011 US Renal Data System annual report ${ }^{1}$ showed slightly decreasing all-cause admission rates: from 1.97 per person-year in 2001 to 1.90 per person-year in 2007. Those rates are higher than the rates we are reporting (1.53 per person-year in 2001 and 1.18 per person-year in 2007), but comparisons should be made with caution because dialysis populations differ substantially between countries. ${ }^{1,8,18}$ Among patients on hemodialysis in 2007, infection-related and cardiovascular-related admission rates were also higher in the US than in our study ( 0.46 per person-year v. 0.19 per person-year for infection-related admissions and 0.56 per person-year v. 0.24 per person-year for cardiovascular-related admissions). ${ }^{1}$ Both rates were relatively stable between 2001 and 2007.

Few studies have evaluated hospital admission rates by length of time on dialysis. The US Renal Data System annual report ${ }^{1}$ suggests that admission rates are higher in the first year of dialysis. Another study showed that more than $50 \%$ of patients initiating dialysis are admitted to hospital in the first 100 days of dialysis. ${ }^{2}$ Our results contribute to these findings: admission rates decreased most rapidly over the first year, and continued to decrease for at least 4 years after initiation of dialysis. During the first year of dialysis, patients are believed to be at greater risk of morbidity and mortality than in subsequent years, ${ }^{1}$ possibly because of greater use of catheters in that period or adjustment of dialysis regimens. ${ }^{19}$ This phenomenon may also be explained by survival effect: patients with severe concurrent conditions will die early, whereas healthier patients remain on dialysis longer. Because patients with more comorbidities are admitted to hospital more often, rates of admission would be higher in the first years when those patients are still in the cohort.

Why infection-related admissions to hospital did not decrease between 2001 and 2007 remains unclear and should be the focus of further studies. The lack of decrease may be

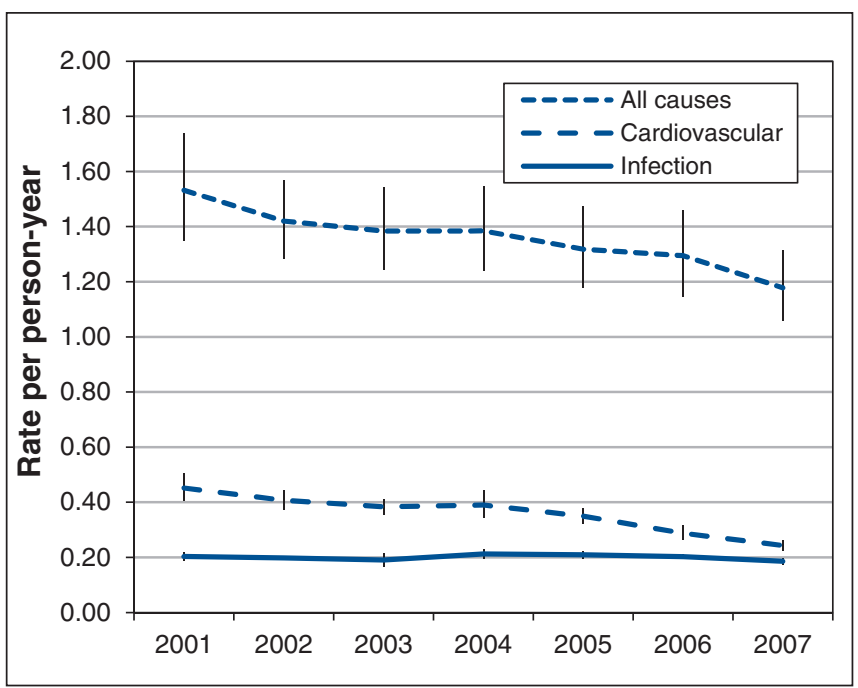

Figure 2: Unadjusted rates of admission to hospital among patients on dialysis, 2001-2007. Error bars $=95 \%$ confidence intervals.

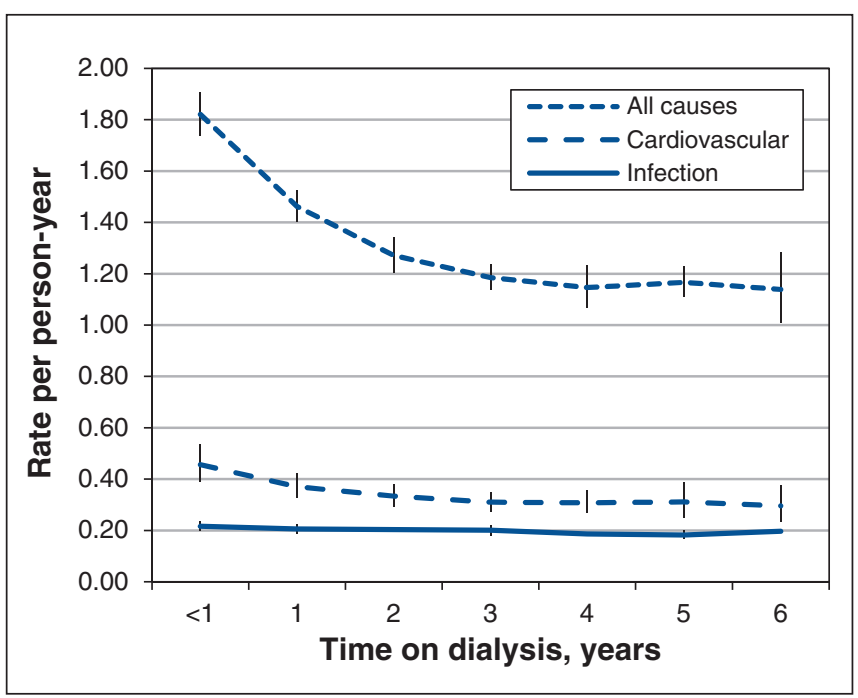

Figure 3: Unadjusted rates of admission to hospital among patients on dialysis, by length of time on dialysis. Error bars $=95 \%$ confidence intervals. 
explained by increasing prevalence of diabetes and an increasing use of catheters in the long-term dialysis population. ${ }^{8,10,19}$ Although infections may be hard to prevent, these factors are modifiable, and intervention strategies may help reduce infection rates. The decreasing trend in all-cause hospital admissions over time may be explained by the fact that some interventions formerly performed in hospital, such as vascular access and cardiovascular procedures, are now done in an outpatient setting. The decreasing trend in cardiovascular-related admissions may be a result of more aggressive treatment of cardiovascular risk factors, such as hyperlipidemia and hypertension.

\section{Strengths and limitations}

Our study's strengths included the ability to follow patients during the first 90 days after dialysis initiation, a high-risk period not often covered in other data sets. In addition, a universal health care setting allowed minimal selection bias.

As in most retrospective studies, our study has several limitations. First, because we relied on claims and ICD-9 and ICD-10 codes to identify patients on dialysis, we may have missed some patients on long-term dialysis. However, the validity of identifying patients on dialysis through procedure billing claims in the Régie de l'assurance maladie du Québec is thought to be high, because such patients receive multiple treatments. Unfortunately, we had no access to data before 1999 . However, excluding patients who initiated dialysis before that date yielded results similar to those for all patients in the study cohort. Because of the prohibitive numbers of strata, we were not able to adjust for age and sex in addition to length of time on dialysis. Adjustment using a multivariable model would overcome this issue, but it would provide an estimate of the trend only, not actual rates. Nevertheless, age and sex distribution did not change significantly during the observed period, and age- and sex-adjusted rates were almost identical to unadjusted rates. Although many factors may change over time and with length of time on dialysis, the goal of this analysis was to evaluate the resulting effect of those changes on infection-related hospital admissions based on length of time on dialysis. Adjusting for intermediate factors, such as comorbidities, could take away the effect of length of time on dialysis. Further studies are needed to investigate interactions between length of time on dialysis and rates of infectionrelated hospital admission.

\section{Conclusion}

We found a decreasing trend in all-cause and cardiovascularrelated rates of admission to hospital among patients on longterm dialysis, independent of age, sex and length of time on dialysis. In contrast, the rate of infection-related hospital admissions remained stable over the period of the study, which meant that the proportion of hospital admissions attributable to infection increased from $13.1 \%$ to $16.1 \%$. Although both all-cause and cardiovascular-related admission rates decreased with length of time on dialysis, infectionrelated admission rates remained stable. Understanding the epidemiology of infection-related hospital admissions among patients receiving long-term dialysis may inform future studies on prevention of this common and serious outcome.

\section{References}

1. US Renal Data System. USRDS 2011 annual data report: atlas of chronic kidney disease and end-stage renal disease in the United States. Bethesda (MD): National Institutes of Health, National Institute of Diabetes and Digestive and Kidney Diseases; 2011.

2. Quinn MP, Cardwell CR, Rainey A, et al. Patterns of hospitalisation before and following initiation of haemodialysis: a 5 year single centre study. Postgrad Med 7 2011;87:389-93.

3. Chavers BM, Solid CA, Gilbertson DT, et al. Infection-related hospitalization rates in pediatric versus adult patients with end-stage renal disease in the United States. F Am Soc Nephrol 2007;18:952-9.

4. Rayner HC, Pisoni RL, Bommer J, et al. Mortality and hospitalization in haemodialysis patients in five European countries: results from the Dialysis Outcomes and Practice Patterns Study (DOPPS). Nephrol Dial Transplant 2004; 19:108-20.

5. Allon M, Depner TA, Radeva M, et al. Impact of dialysis dose and membrane on infection-related hospitalization and death: results of the HEMO Study. $7 \mathrm{Am} \mathrm{Soc}$ Nephrol 2003;14:1863-70.

6. Allon M, Radeva M, Bailey J, et al. The spectrum of infection-related morbidity in hospitalized haemodialysis patients. Nephrol Dial Transplant 2005;20:1180-6.

7. Dalrymple LS, Mohammed SM, Mu Y, et al. Risk of cardiovascular events after infection-related hospitalizations in older patients on dialysis. Clin $7 \mathrm{Am} \mathrm{Soc}$ Nephrol 2011;6:1708-13.

8. Treatment of end-stage organ failure in Canada, 2001 to 2010. Ottawa (ON): Canadian Institute for Health Information; 2012.

9. Canaud B, Tong L, Tentori F, et al. Clinical practices and outcomes in elderly hemodialysis patients: results from the Dialysis Outcomes and Practice Patterns Study (DOPPS). Clin 7 Am Soc Nephrol 2011;6:1651-62.

10. Ethier J, Mendelssohn DC, Elder SJ, et al. Vascular access use and outcomes: an international perspective from the Dialysis Outcomes and Practice Patterns Study. Nephrol Dial Transplant 2008;23:3219-26.

11. Levy AR, Mayo NE, Grimard G. Rates of transcervical and pertrochanteric hip fractures in the province of Quebec, Canada, 1981-1992. Am 7 Epidemiol 1995;142:428-36.

12. Mayo NE, Goldberg MS, Levy AR, et al. Changing rates of stroke in the province of Quebec, Canada: 1981-1988. Stroke 1991;22:590-5.

13. Jollis JG, Ancukiewicz M, DeLong ER, et al. Discordance of databases designed for claims payment versus clinical information systems. Implications for outcomes research. Ann Intern Med 1993;119:844-50.

14. Tamblyn R, Reid T, Mayo N, et al. Using medical services claims to assess injuries in the elderly: sensitivity of diagnostic and procedure codes for injury ascertainment. 7 Clin Epidemiol 2000;53:183-94.

15. Zou G. A modified poisson regression approach to prospective studies with binary data. Am 7 Epidemiol 2004;159:702-6.

16. Rothman KJ, Greenland S, Lash TL. Modern epidemiology. 3rd ed. Philadelphia (PA): Lippincott Williams \& Wilkins; 2008.

17. Koepsell TD, Weiss NS. Epidemiologic methods: studying the occurrence of illness. New York (NY): Oxford University Press; 2003.

18. Goodkin DA, Young EW, Kurokawa K, et al. Mortality among hemodialysis patients in Europe, Japan, and the United States: case-mix effects. Am 7 Kidney Dis 2004;44(Suppl 2):16-21.

19. Lafrance JP, Iqbal S, Lelorier J, et al. Vascular access-related bloodstream infections in First Nations, community and teaching Canadian dialysis units, and other centre-level predictors. Nephron Clin Pract 2010;114:c204-12.

Affiliations: Centre de recherche Hôpital Maisonneuve-Rosemont (Lafrance, Elftouh); Département de médecine (Lafrance, Vallée), Université de Montréal; Service de néphrologie (Lafrance, Laurin, Vallée), Hôpital Maisonneuve-Rosemont; Department of Medicine (Rahme, Iqbal), McGill University; Research Institute (Rahme), McGill University Health Centre; Division of Nephrology (Iqbal), McGill University Health Centre, Montréal, Que.

Contributors: This manuscript was coauthored by Jean-Philippe Lafrance (design, analysis and interpretation of data, drafting of the article), Elham Rahme (design, revision of the article), Sameena Iqbal (design, revision of the article), Naoual Elftouh (analysis and interpretation of data, revision of the article), Louis-Philippe Laurin (design, revision of the article) and Michel Vallée (design, revision of the article). All of the authors approved the final version of the manuscript.

Funding: This work was supported by a Fonds de la recherche en santé du Québec operating grant. Jean-Philippe Lafrance was supported by a KRESCENT (Kidney Research Scientist Core Education and National Training Program) New Investigator Award.

Supplemental information: For reviewer comments and the original submission of this manuscript, please see www.cmajopen.ca/content/2/2 /E109/suppl/DC1 